\title{
Dicer ablation promotes a mesenchymal and invasive phenotype in bladder cancer cells
}

\author{
ALAN P. LOMBARD ${ }^{1-3}$, REBECCA M. LIM ${ }^{1}$, RACHEL M. NAKAGAWA ${ }^{1}$, KATHLEEN D. VIDALLO ${ }^{1}$, \\ STEPHEN J. LIBERTINI ${ }^{1,3}$, ALEXANDER J. PLATERO ${ }^{1}$ and MARIA MUDRYJ ${ }^{1,3}$ \\ ${ }^{1}$ Department of Medical Microbiology and Immunology, ${ }^{2}$ Biochemistry, Molecular, Cellular, and \\ Developmental Biology Graduate Group and Biotechnology Program, University of California, Davis; \\ ${ }^{3}$ Veterans Affairs-Northern California Health Care System, Mather, CA, USA
}

Received January 24, 2015; Accepted March 16, 2015

DOI: $10.3892 /$ or.2015.4117

\begin{abstract}
Dicer expression is frequently altered in cancer and affects a wide array of cellular functions acting as an oncogene or tumor suppressor in varying contexts. It has been shown that Dicer expression is also deregulated in urothelial cell carcinoma of the bladder (UCCB) but the nature of this deregulation differs between reports. The aim of the present study was to gain a better understanding of the role of Dicer in bladder cancer to help determine its contribution to the disease. The results showed that Dicer transcript levels were decreased in UCCB tumor tissues as compared to normal tissues, suggesting that Dicer is a tumor suppressor. However, consistent with previous results, we demonstrated that knockdown of Dicer decreases cell viability and increases the induction of apoptosis, suggesting that Dicer is an oncogene. To resolve this discrepancy, we assessed the effects of decreased Dicer expression on epithelial-to-mesenchymal transition, migration and invasion. We showed that decreased Dicer levels promoted a mesenchymal phenotype and increased migration. Additionally, the results showed that Dicer protein ablation leads to increased cell invasion, higher levels of matrix metalloproteinase-2, and decreased levels of key miRNAs shown to inhibit invasion. The results of this study suggest that decreased Dicer levels may portend a more malignant phenotype.
\end{abstract}

Correspondence to: Dr Maria Mudryj, Department of Medical Microbiology and Immunology, University of California, 1 Shields Avenue, Tupper Hall 3147, Davis, CA 95616, USA

E-mail: mmudryj@ucdavis.edu

Abbreviations: SDS-PAGE, sodium dodecyl sulfate-polyacrylamide gel electrophoresis; PBS-T, 0.1\% Tween-phosphate-buffered saline; HRP, horseradish peroxidase; PARP, poly(ADP-ribose) polymerase; qPCR, quantitative polymerase chain reaction; FBS, fetal bovine serum; siRNA, small-interfering RNA

Key words: bladder cancer, Dicer, invasion, proliferation, apoptosis, mesenchymal, epithelial-to-mesenchymal transition

\section{Introduction}

Dicer is a $219-\mathrm{kDa}$ protein that functions to cleave doublestranded RNA (1). It is a key component of RNAi pathways and aids in the biogenesis of miRNAs and siRNAs, rendering it a major regulatory molecule with wide-reaching pleiotropic effects (1). Dicer function can be linked to several cell processes and its dysregulation and altered expression has been studied in several malignancies (1). It affects several hallmarks of cancer including proliferation, evasion of cell death, and migration and invasion and it has been shown to play a role as an oncogene or tumor suppressor depending on the context. In support of an oncogenic role for Dicer, it has been shown to be upregulated in prostate and colorectal cancers $(2,3)$. Furthermore, inhibition of Dicer expression was shown to attenuate prostate cancer cell growth by promoting cell cycle arrest and induction of apoptosis (4). Downregulation of Dicer in ovarian, lung, nasopharyngeal and kidney cancers supports its role as a tumor suppressor (5-8). In ovarian cancer, it was demonstrated that the knockdown of Dicer led to increased cell proliferation and migration (9).

The status of Dicer in urothelial cell carcinoma of the bladder(UCCB) has been studied; however, the reported results are inconsistent. In a study by Catto et al, it was demonstrated that Dicer mRNA expression was downregulated 7.4-fold in UCCB tissues as compared to normal tissues (10). These data were supported in a study by Wu et al which assessed Dicer expression at the mRNA and protein levels (11). In contrast to these studies, Han et al demonstrated using qPCR on 40 UCCB samples and matched controls that Dicer was upregulated in this disease (12).

The function of Dicer in UCCB has also been investigated. Current data suggest an oncogenic role for this protein despite studies showing that it has a downregulated expression pattern. It was demonstrated that siRNA-mediated knockdown of Dicer inhibited proliferation of the T24 UCCB cell line (13). In addition to analyzing the expression of Dicer, Han et al showed that inhibition of Dicer attenuated proliferation and induced apoptosis in T24 and 5637 UCCB cells (12). In contrast to this suggested oncogenic role, it has been shown that Dicer negatively regulates migration and invasion in other contexts such as ovarian cancer $(9,14)$. This observation suggests that 
Dicer serves as a tumor suppressor that regulates the metastatic cascade. However, whether Dicer affects migration and invasion in UCCB remains to be investigated.

In the present study, we aimed to gain a better understanding of the role Dicer plays in UCCB. We hypothesized that Dicer has a dual nature in this disease possessing attributes of both an oncogene and a tumor suppressor. We investigated Dicer expression in UCCB tissues and we found that the attenuation of Dicer protein levels led to a decrease in cell viability in part through induction of apoptosis. Additionally, we found that a decrease in Dicer expression promoted a mesenchymal phenotype and increased invasion and the expression of matrix metalloproteinase-2 (MMP-2). We also showed that several miRNAs shown to be negatively associated with an invasive phenotype were downregulated when Dicer was knocked down. Thus, we demonstrated novel functions for Dicer in UCCB. The present study aimed to reconcile contradictions in the field by suggesting that the downregulation of Dicer may portend a more invasive and therefore metastatic phenotype.

\section{Materials and methods}

Tissues, cell lines and transfections. UCCB tissue samples and normal tissue control samples were obtained from the UC Davis Cancer Center Biorepository. SV-HUC-1, T24, TCCSUP, J82 and UM-UC-3 cells were generous gifts from Dr Sweeney and Dr De Vere White (UC Davis). The cell lines were cultured in $10 \%$ fetal bovine serum (FBS) RPMI-1640 supplemented with glutamine, penicillin and streptomycin at $37^{\circ} \mathrm{C}$ and $5 \%$ $\mathrm{CO}_{2}$. Transfections were carried out using Lipofectamine RNAiMAX (Invitrogen-Life Technologies, Carlsbad, CA, USA) at an oligonucleotide concentration of $50 \mathrm{nM}$. siRNAs used were: ON-TARGET plus control pool (non-targeting), and Dicer (custom sequence, AAGGCUUACCUUCUCC AGGCUUU) (both from Dharmacon, Lafayette, CO, USA).

Cell viability assays. T24, TCCSUP, J82 and UM-UC-3 cells were plated at 10,000-25,000 cells/well in 24-well plates. The cells were transfected with either control non-targeting scrambled oligonucleotides or Dicer targeting siRNA and viability was assessed at 48 and $96 \mathrm{~h}$ using Cell Counting Kit-8 (CCK-8) viability detection reagent (Dojindo). Conditions were plated in triplicate. Data were presented as mean \pm standard deviation.

Western blotting. Whole cell lysates were subjected to sodium dodecyl sulfate-polyacrylamide gel electrophoresis (SDS-PAGE) and transferred to $0.2-\mu \mathrm{m}$ nitrocellulose membranes. The membranes were blocked in 5\% milk in $0.1 \%$ Tween-phosphate-buffered saline (PBS-T) and were allowed to incubate with primary antibody overnight at $4^{\circ} \mathrm{C}$. The following day, the membranes were incubated with secondary antibody conjugated to horseradish peroxidase (HRP), developed using the WesternBright Sirius kit (Advansta, Menlo Park, CA, USA) and visualized with a Konica Minolta SRX-101A developer or a Li-Cor C-DiGit Scanner. Tubulin, actin or Ponceau S stain served as loading controls. The antibodies used were: poly(ADP-ribose) polymerase (PARP), E-cadherin, N-cadherin, MMP-2 (Cell Signaling Technology, Inc., Danvers, MA, USA), Dicer (Abcam, Cambridge, UK), tubulin (Thermo Fisher Scientific, Rockford, IL, USA) and actin (Millipore, Billerica, MA, USA).

Quantitative PCR ( $q P C R)$. RNA was extracted using either the RNeasy kit (Qiagen, Hilden, Germany) or the mirVana miRNA Isolation kit (Ambion-Life Technologies, Carlsbad, CA, USA) and reverse transcribed into cDNA using either the QuantiTect reverse transcription kit or the miScript II RT kit (both from Qiagen). qPCR was performed using the KAPA SYBR FAST Universal qPCR kit (Kapa Biosystems) or the miScript PCR kit (Qiagen) on a ViiA 7 Real-Time PCR System (Applied Biosystems). Data were analyzed using the efficiency-corrected $\Delta \mathrm{Ct}$ method. The primers used were: $\mathrm{miR}-205$, miR-31, miR200a, miR-200b, miR-200c, miR-148a, miR-149 and miR-106b miScript primer assays (Qiagen); HPRT, F-GCCAGA CTTTGTTGGATTTG and R-CTCTCATCTTAGGCTTTG TATTTTG; Dicer, F-TCCACGAGTCACAATCAACACGG and R-GGGTTCTGCATTTAGGAGCTAGATGAG.

Invasion and migration assays. Invasion and migration were assessed using Transwell assays with or without Matrigel membrane coating, respectively (Cell Biolabs, Inc., San Diego, CA, USA; CBA-101-C). Cell seeding was normalized using a parallel viability assay as described below. Briefly, the cells were plated and then treated $24 \mathrm{~h}$ later with a control non-targeting oligonucleotide or Dicer targeting siRNA. After 48 h, 10\% FBS containing RPMI-1640 was placed in the bottom chambers of the wells and the cells were seeded into the top chambers in media with no serum. Twenty-four hours later, invaded/ migrated cells were removed, lysed and read using a fluorescent plate reader at $480 / 520 \mathrm{~nm}$. Invasion/migration was normalized to cell viability from a parallel assay utilizing the cells from the same suspensions used to seed the invasion/migration assays. Viability was read using the CCK-8 reagent as previously described. The conditions were carried out in triplicate. Data were presented as mean \pm standard deviation.

Statistical analysis. Data were presented as mean \pm standard deviation. A two-tailed, two-sampled equal variance Student's $\mathrm{t}$-test was used to assess the differences between the samples. $\mathrm{P} \leq 0.05$ was considered to indicate a statistically significant result.

\section{Results}

Expression of Dicer is downregulated in UCCB tumor tissues. Previous studies have demonstrated that Dicer expression is altered in the context of UCCB, albeit the nature of this expression alteration is unclear (10-12). Using qPCR, we examined the expression of Dicer in UCCB tumor samples. We found a statistically significant decrease in Dicer expression in tumor tissues as compared to normal tissues (Fig. 1A). Thus, we hypothesized that Dicer may have an unidentified tumor-suppressor role in this disease. Additionally, we performed western blot analysis to define the Dicer protein levels in the T24, TCCSUP, J82 and UM-UC-3 UCCB cell lines and we found that Dicer expression was variable (Fig. 1B).

Knockdown of Dicer in UCCB cell lines inhibits proliferation and induces apoptosis. We aimed to better understand how 


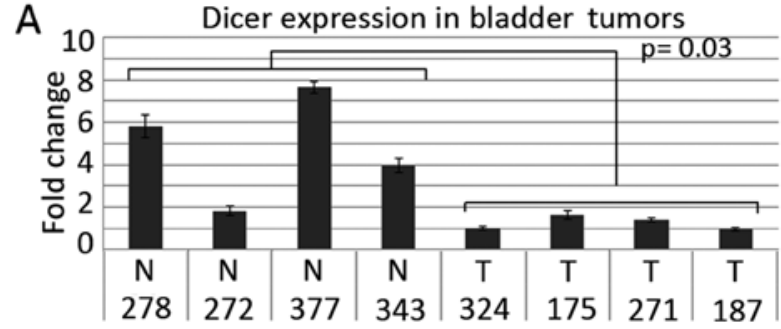

B

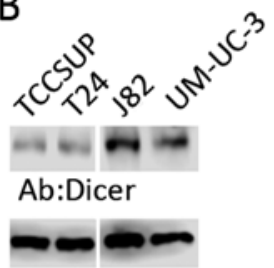

Ab: Actin

Dicer protein normalized to actin

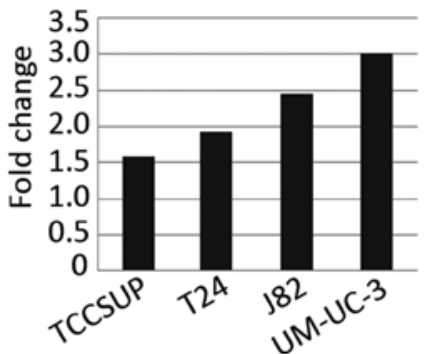

Figure 1. Expression of Dicer in UCCB. (A) qPCR was used to assess Dicer transcript levels in four UCCB tumor samples vs. four normal tissue samples. (B) Western blot analysis shows Dicer protein levels in the TCCSUP, T24, J82 and UM-UC-3 UCCB cell lines. Actin served as a loading control. Densitometry demonstrates the fold-change differences in Dicer protein expression shown in the right panel of (B) normalized to actin protein expression. $\mathrm{N}$, normal tissue; $\mathrm{T}$, tumor tissue. Numbers represent patient labels. p, p-value; Ab, antibody used for western blot analyses. Error bars are the standard deviation. UCCB, urothelial cell carcinoma of the bladder.

Dicer functions in UCCB by knocking down Dicer in the four cell lines mentioned above and assessing viability (Fig. 2A). We found that a decreased expression of Dicer led to the attenuation of cell growth in all the cell lines except for J82 (Fig. 2B). The results are consistent with previous studies that show that decreased Dicer impairs proliferation $(12,13)$. The reason for J82 cells being refractory to this treatment is unclear. However, this result highlights known heterogeneity found in different tumors. These data suggest that in certain contexts, Dicer increases cell viability and plays an oncogenic role.

We assessed whether the decrease in cell viability in response to Dicer knockdown was due to an increase in apoptosis (Fig. 2C). Using western blot analysis to detect cleaved PARP, we demonstrated that a decreased Dicer expression induced apoptosis in T24 and UM-UC-3 cells. As with the viability assay above, we observed no change in J82. Notably, no change was observed in TCCSUP, suggesting that decreased viability previously noted in this cell line may not be due to apoptosis in these cells. Alternatively, apoptosis in this cell line may not result in PARP cleavage.

Decreased Dicer expression promotes a mesenchymal phenotype. Recent evidence has shown that lower levels of Dicer in breast and ovarian cancers are associated with an epithelial-tomesenchymal transition (EMT), a mesenchymal phenotype, and leads to increased motility $(9,15,16)$. Since we and other investigators have shown that Dicer can be downregulated in UCCB, we hypothesized that Dicer may function similarly in this context and thus is also able to serve a tumor-suppressor role $(10,11)$. To assess a possible role in EMT for Dicer, we examined the expression of the mesenchymal marker

A
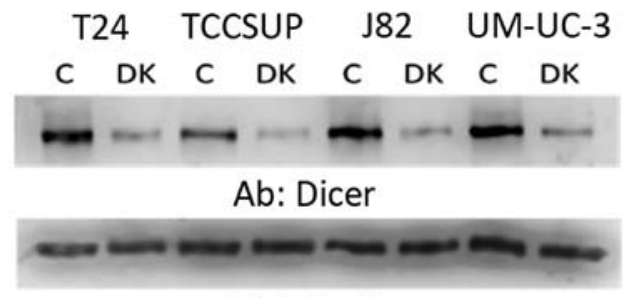

Ab: Tubulin

B
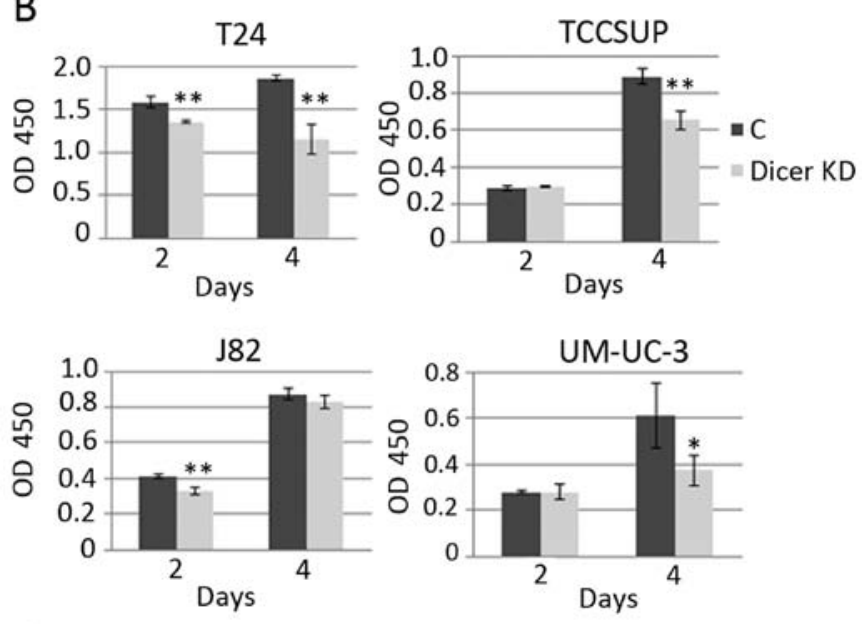

C

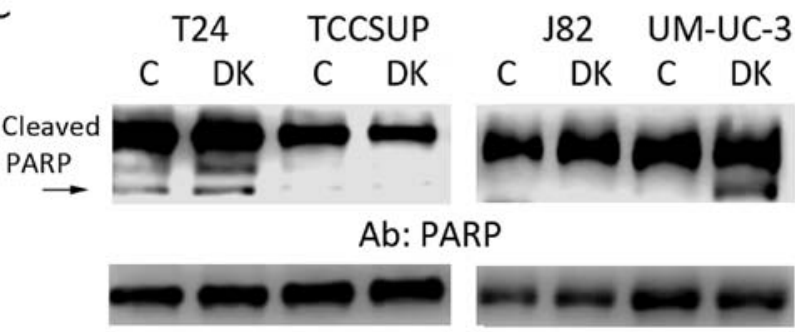

Ab: Tubulin

Figure 2. Dicer knockdown attenuates viability of UCCB cell lines. (A) Western blot analyses for Dicer demonstrate the efficacy of Dicer knockdown via siRNA treatment. Tubulin served as a loading control. (B) Viability assays demonstrate the effect of Dicer knockdown on the T24, TCCSUP, J82 and UM-UC-3 UCCB cell lines. (C) Western blot analyses for PARP cleavage were used to assess the induction of apoptosis in response to Dicer knockdown. Tubulin served as a loading control. C, control oligonucleotide; DK, siRNA targeting Dicer; Dicer KD, Dicer knockdown; Ab, antibody used for western blotting. Error bars are the standard deviation. ${ }^{*} p \leq 0.05$ and ${ }^{* *} p \leq 0.01$. UCCB, urothelial cell carcinoma of the bladder; PARP, poly(ADP-ribose) polymerase.

$\mathrm{N}$-cadherin and the epithelial marker E-cadherin (Fig. 3). We found that Dicer knockdown led to an increase in N-cadherin expression in T24 and TCCSUP cells. Depletion of Dicer did not cause an alteration of $\mathrm{N}$-cadherin expression in $\mathrm{J} 82$ cells. In UM-UC-3 cells, we detected a very low expression of N-cadherin which appeared to decrease with Dicer knockdown. E-cadherin was detected in TCCSUP cells but not in T24, J82 and UM-UC-3 cells. However, E-cadherin did not change in response to Dicer knockdown in TCCSUP cells. Additionally, Dicer depletion did not induce the expression of E-cadherin in T24, J82 and UM-UC-3 cells (data not shown). These data indicate that decreased Dicer expression promotes a more mesenchymal phenotype in specific cell contexts via the upregulation of N-cadherin. However, Dicer downregulation failed to promote complete EMT. 


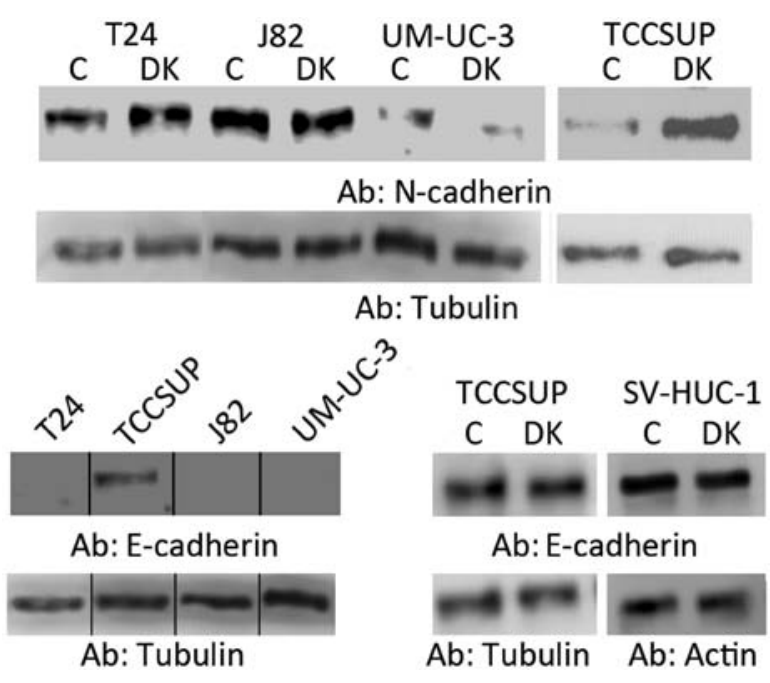

Figure 3. Effects of Dicer knockdown on mesenchymal phenotype. Western blot analyses for the mesenchymal marker $\mathrm{N}$-cadherin and the epithelial marker E-cadherin were used to assess EMT in response to Dicer knockdown in the T24, TCCSUP, J82 and UM-UC-3 UCCB cell lines and the immortalized SV-HUC-1 urothelial cell line. Tubulin or actin served as a loading control. C, control oligonucleotide; DK, siRNA targeting Dicer; Ab, antibody used for western blotting. EMT, epithelial-to-mesenchymal transition; UCCB, urothelial cell carcinoma of the bladder.

Additionally, we determined whether Dicer knockdown promotes a mesenchymal phenotype in an immortalized non-transformed cell line. Using SV-HUC-1 cells, we again assessed EMT marker expression and found that the attenuation of Dicer did not reduce E-cadherin expression (Fig. 3). $\mathrm{N}$-cadherin was not detected in these cells and its expression was not induced by Dicer knockdown (data not shown).

Dicer knockdown increases cell migration and invasion and MMP-2 expression. A study by Nieman et al demonstrated that $\mathrm{N}$-cadherin expression is more indicative of a motile and invasive phenotype rather than a loss of E-cadherin (17). They also showed that a forced expression of $\mathrm{N}$-cadherin in E-cadherin-expressing cells promoted motility and invasion despite the presence of E-cadherin (17). Thus, we conclude from our EMT data that decreased Dicer expression can promote mesenchymal properties and therefore may promote a more motile and invasive phenotype via upregulation of $\mathrm{N}$-cadherin in T24 and TCCSUP cells.

Using transwell migration assays, we determined whether decreased Dicer expression promotes migration and invasion (Fig. 4A and B). Because we hoped to augment function, we wanted to choose the line that would allow us to detect the most change. Based on previous studies of the invasive propensities of UCCB cell lines, we decided that TCCSUP cells would be best suited for these studies since they exhibit a low invasive capability compared to T24 (18). Furthermore, TCCSUP cells exhibited the most robust induction of $\mathrm{N}$-cadherin expression in response to Dicer knockdown. We also used SV-HUC-1 in the present study (also shown to have low invasiveness) to further assess whether Dicer inhibition regulates motility and whether it may function to promote malignancy in a non-transformed context (19). Our data demonstrated that Dicer knockdown increased the migration of TCCSUP cells but only potentiated a trend towards increased motility in the SV-HUC-1 cells
A
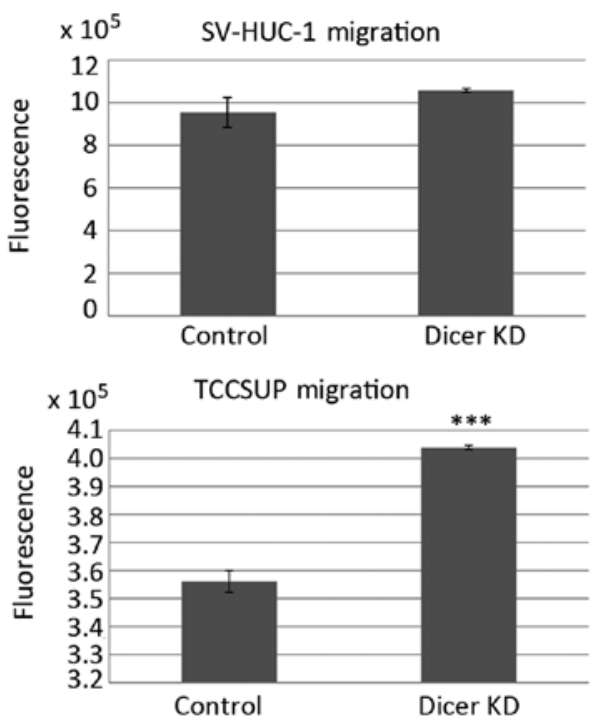

B
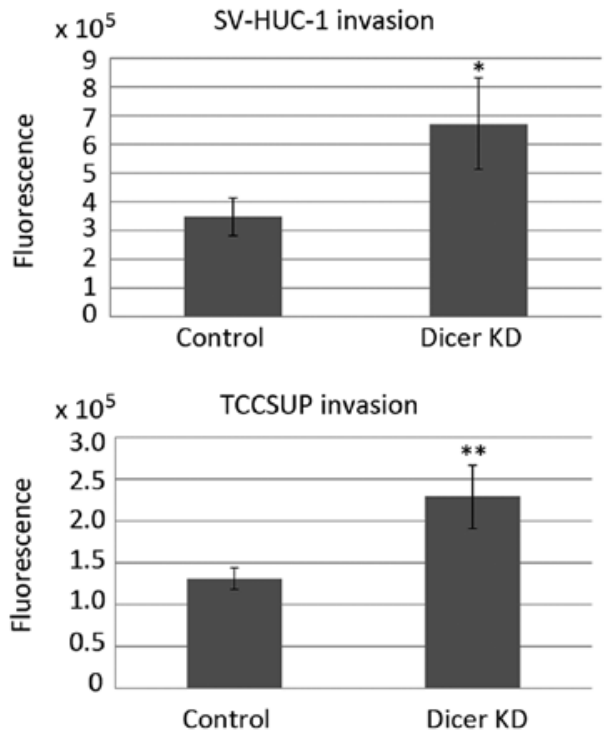

C

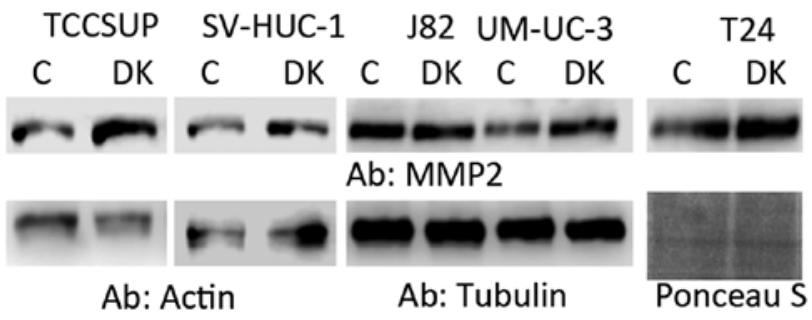

Figure 4. Effects of Dicer knockdown on migration, invasion and MMP-2 expression. (A) Transwell migration assays demonstrated the effect of Dicer knockdown on cell motility in SV-HUC-1 and TCCSUP cells. (B) Matrigelcoated transwell invasion assays demonstrated the effect of Dicer knockdown on cell invasion in SV-HUC-1 and TCCSUP cells. (C) Western blot analyses demonstrate the effect of Dicer knockdown on MMP-2 in TCCSUP, SV-HUC-1, J82, UM-UC-3 and T24 cells. Actin, tubulin and Ponceau S served as loading controls. C, control oligonucleotide; DK, siRNA targeting Dicer; Dicer KD, Dicer knockdown; Ab, antibody used for western blotting. Error bars are the standard deviation. ${ }^{*} \mathrm{p} \leq 0.05,{ }^{* *} \mathrm{p} \leq 0.01,{ }^{* * *} \mathrm{p} \leq 0.001$. MMP-2, matrix metalloproteinase-2.

$(\mathrm{p}=0.06)($ Fig. 4A). We conclude from these studies that Dicer can play a role in regulating motility of UCCB cells.

Studies have also shown that decreased levels of Dicer led to increased cell invasion (20). Thus, in addition to assessing 

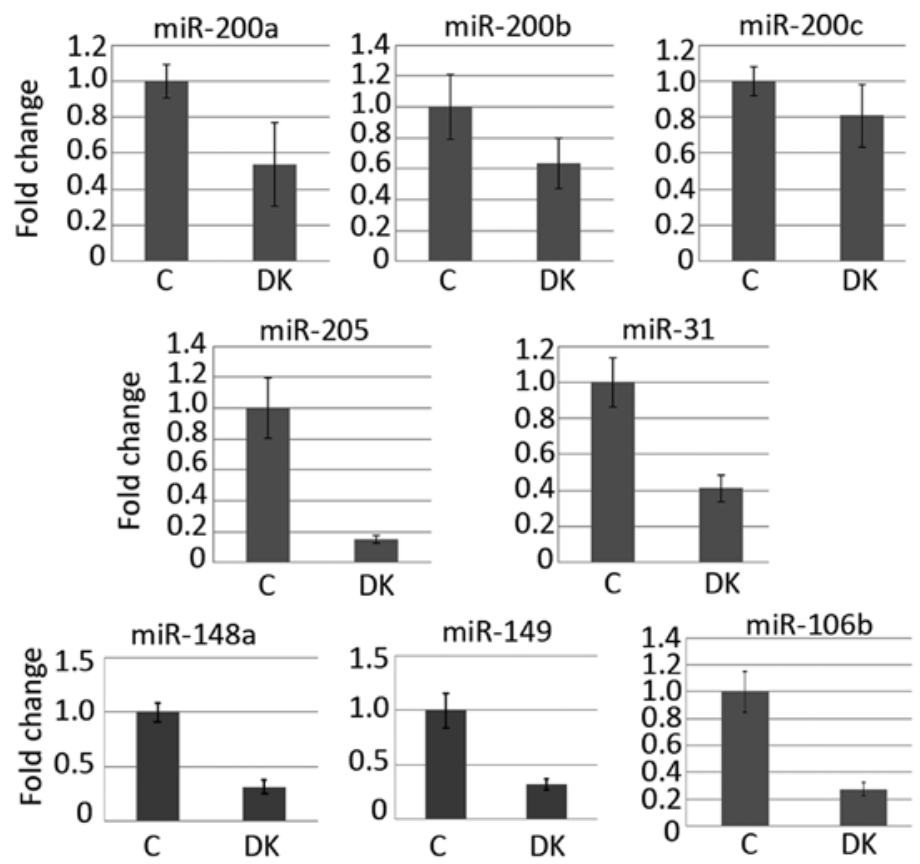

Figure 5. Dicer knockdown attenuates expression of invasion-associated miRNAs. The knockdown of Dicer led to a decreased expression of invasion-associated miRNAs: miR-200a, miR-200b, miR-200c, miR-205, miR-31, miR-148a, miR-149 and miR-106b. Error bars are the standard deviation. C, control oligonucleotide; DK, siRNA targeting Dicer.

migration, we examined whether Dicer regulates the invasive ability of UCCB cells (Fig. 4B). Using a matrigel-coated transwell assay, we demonstrated that Dicer knockdown increases invasion almost 2-fold in the TCCSUP and SV-HUC-1 cells suggesting that lower levels of Dicer may potentiate a more metastatic phenotype. Western blot analyses demonstrated that Dicer knockdown also led to increased levels of MMP-2 in the two cell lines (Fig. 4C). Additionally, we showed that Dicer knockdown increased the expression of MMP-2 in T24 and UM-UC-3 cells, but not in J82 cells Fig. 4C). It has been shown that MMP-2 expression and activity are associated with increased stage and invasiveness of bladder cancer, respectively $(21,22)$. These data suggest that decreased Dicer may increase the invasive abilities of UCCB cells and demonstrate a mechanism by which Dicer plays a tumor-suppressor role.

Decreased Dicer expression leads to attenuated expression of invasion-associated miRNAs. We used qPCR to assess the expression of miRNAs associated with a motile/invasive phenotype (Fig. 5). Based on Dicer's known functions, we hypothesized that miRNAs may be ultimately responsible for the effects observed when Dicer is knocked down. In a study by Wszolek et al, a panel of invasion-associated miRNAs was determined using qPCR comparing 31 invasive UCCB lesions to 26 non-invasive UCCB lesions (23). From this panel, 5 miRNAs were selected to investigate in response to Dicer knockdown. We selected the well-characterized miR-200a/b/c in addition to miR-31, which has been shown to negatively correlate with UCCB patient progression and mortality, and miR-205 which was demonstrated to have strong discriminatory power in distinguishing invasive UCCB tumors from non-invasive UCCB tumors (24-26). Wzsolek et al showed that the overexpression of these miRNAs in UM-UC-3 cells attenu- ated invasion using a transwell matrigel-coated membrane assay (23). In response to Dicer knockdown, we found small decreases in the levels of miR-200a/b/c (Fig. 5). However, we found a $>5$-fold reduction in miR-205 levels and a $\sim 2$-fold decrease in miR-31 expression (Fig. 5). These data along with the study by Wzsolek suggested that decreased Dicer expression may potentiate cell invasion via decreased expression of miRNAs (23). However, we hypothesized that decreased Dicer could lead to global downregulation of miRNAs. Using qPCR, we assessed the expression of additional miRNAs (miR-148a, miR-149 and miR-106b) associated with invasion in other contexts and found these were also downregulated in response to attenuated Dicer levels (Fig. 5) (27-29). Our data suggest that pan miRNA expression may be decreased along with decreased Dicer levels and that the global downregulation of miRNAs promotes an invasive phenotype.

\section{Discussion}

The expression of Dicer in UCCB is unclear and its function in UCCB tumorigenesis is only partially understood. The present study investigated the possible bases of differing results while expanding our current understanding of Dicer in these tumors. We demonstrated that Dicer expression was decreased in UCCB tumor tissue using qPCR, a result that is consistent with previous reports $(10,11)$. However, a study by Han et al demonstrated an increased expression of Dicer in UCCB (12). We hypothesized that Dicer expression is variable in UCCB tumors and may function in distinct ways.

Two prior reports indicate that Dicer is oncogenic $(12,13)$. Our data are consistent with these findings demonstrating that attenuation of Dicer in UCCB cell lines results in decreased viability. However, J82 cells proved refractory to this treatment. We also showed that Dicer knockdown induced cell 
death in T24 and UM-UC-3 cells, but this was not evident in J82 or TCCSUP cells. Our results emphasize the heterogeneity of cancer and demonstrate that the importance of Dicer in cellular viability varies with context. Collectively, these data suggest that Dicer plays an oncogenic role by promoting cell viability and inhibiting cell death. However, our and previous studies have demonstrated the downregulation of Dicer in UCCB, suggesting that Dicer also functions as a tumor suppressor $(10,11)$. We resolved this contradiction by assessing the role of Dicer in regulating motility and invasion.

Several lines of evidence have shown that Dicer is involved in elements of the metastatic cascade $(9,15,16)$. We hypothesized that Dicer downregulation in UCCB may promote EMT and increase migration and invasion. Our data demonstrates that Dicer knockdown promoted a mesenchymal phenotype by inducing N-cadherin expression in T24 and TCCSUP cells. While decreased Dicer expression failed to promote complete EMT, a previous study has shown that the expression of N-cadherin is more indicative of a motile and invasive phenotype (17).

We next assessed migration and found that Dicer knockdown led to an increase in migration in TCCSUP cells. A trend towards enhancement of migration in SV-HUC-1 cells was identified. These data suggest that in the context of UCCB, Dicer is able to enhance migratory propensity. Notably, we found that Dicer knockdown enhanced invasion almost 2-fold in TCCSUP and SV-HUC-1 cells. This phenotypic change was accompanied by an increase in MMP-2 protein expression, suggesting a potential mechanism by which Dicer alters invasiveness. Additionally, decreased Dicer levels led to an increase in MMP-2 in T24 and UM-UC-3 cells, indicating this effect may be widespread in UCCB. We did not detect this change in J82 cells, which were an outlier throughout the present study. J82 cells may have evolved mechanisms that reduce their dependence on Dicer. Our data suggest that decreased Dicer expression may promote a more malignant phenotype by promoting motility and invasion.

The present findings show that the downregulation of Dicer led to decreased levels of key miRNAs known to be negatively associated with invasion in UCCB. However, Dicer knockdown may also affect other miRNAs and could lead to a general downregulation of miRNAs. We assessed the levels of additional miRNAs and found decreased levels of these. This led us to hypothesize that the overall outcome of a widespread downregulation of miRNAs promotes an invasive phenotype in this disease. Consistent with this hypothesis, several studies have correlated the downregulation of Dicer with metastasis and metastatic potential $(5,16,30,31)$. A study by Luo et al in nasopharyngeal cancer showed that lower levels of Dicer led to an overall decrease of most miRNAs (32). This was associated with increased invasion and mobility (32). Iliou et al demonstrated that Dicer impairment led to increased colon cancer cell metastasis and this was associated with a decreased expression of key EMT/invasion miRNAs (miR-200 family, miR-34a, miR-126 and miR-335) (33).

The present study in conjunction with other studies suggests a dual nature for Dicer. It has been postulated that Dicer serves different roles in different contexts and we hypothesized that as cancer progresses, the requirement for Dicer and miRNA expression varies. It is thought that tumors are very heterogeneous and that the invasive edge of a tumor can be quite different than the bulk of the tumor (34). The varying levels of Dicer potentiate different phenotypes at strategic time-points during tumor formation regulating robust proliferation and invasive capacity. Zhang et al showed that Dicer promoted the proliferation of prostate cancer cells (35). However, despite this attribute and an increased expression of Dicer in this disease, it was also shown that relatively lower levels of Dicer promoted a more motile and invasive phenotype (35). In the study by Iliou et al, it was demonstrated that Dicer impairment led to an increase in stemness and an increased capacity for tumor initiation suggesting that Dicer plays a role in cancer stem cells and may be involved in seeding metastases (33). Our data along with previous literature suggest a complex and multifaceted role for Dicer in cancer.

In conclusion, the present study has demonstrated that Dicer has a dual nature in UCCB as it plays the role of an oncogene by promoting proliferation and viability, and the role of a tumor suppressor by inhibiting motility and invasion. We hypothesized that the requirement for Dicer and its role in tumorigenesis may be different at distinct stages of the disease.

\section{Acknowledgements}

We thank Zachary Caudle for his contributions to the present study. The present study is based on a study supported in part by the US Department of Veterans Affairs, Office of Research and Development, Biomedical Laboratory Research Program (VA Merit grant BX001079 to M.M.). The contents of this manuscript do not represent the views of the Department of Veterans Affairs or the United States Government.

\section{References}

1. Foulkes WD, Priest JR and Duchaine TF: DICER1: Mutations, microRNAs and mechanisms. Nat Rev Cancer 14: 662-672, 2014.

2. Chiosea S, Jelezcova E, Chandran U, Acquafondata M, McHale T, Sobol RW and Dhir R: Up-regulation of Dicer, a component of the microRNA machinery, in prostate adenocarcinoma. Am J Pathol 169: 1812-1820, 2006.

3. Faber C, Horst D, Hlubek F and Kirchner T: Overexpression of Dicer predicts poor survival in colorectal cancer. Eur J Cancer 47: 1414-1419, 2011.

4. Bian XJ, Zhang GM, Gu CY, Cai Y, Wang CF, Shen YJ, Zhu Y, Zhang HL, Dai B and Ye DW: Down-regulation of Dicer and Ago2 is associated with cell proliferation and apoptosis in prostate cancer. Tumour Biol 35: 11571-11578, 2014.

5. Ma X, Fan Y, Gao Y, Zhang Y, Huang Q, Ai Q, Ni D, Chen W, Zhang P, Song E, et al: Dicer is down-regulated in clear cell renal cell carcinoma and in vitro Dicer knockdown enhances malignant phenotype transformation. Urol Oncol 32: 46.e9-e17, 2014.

6. Karube Y, Tanaka H, Osada H, Tomida S, Tatematsu Y Yanagisawa K, Yatabe Y, Takamizawa J, Miyoshi S, Mitsudomi T, et al: Reduced expression of Dicer associated with poor prognosis in lung cancer patients. Cancer Sci 96: 111-115, 2005.

7. Guo X, Liao Q, Chen P, Li X, Xiong W, Ma J, Li X, Luo Z, Tang H, Deng M, et al: The microRNA-processing enzymes: Drosha and Dicer can predict prognosis of nasopharyngeal carcinoma. J Cancer Res Clin Oncol 138: 49-56, 2012.

8. Merritt WM, Lin YG, Han LY, Kamat AA, Spannuth WA, Schmandt R, Urbauer D, Pennacchio LA, Cheng JF, Nick AM, et al: Dicer, Drosha, and outcomes in patients with ovarian cancer. N Engl J Med 359: 2641-2650, 2008.

9. Kuang Y, Cai J, Li D, Han Q, Cao J and Wang Z: Repression of Dicer is associated with invasive phenotype and chemoresistance in ovarian cancer. Oncol Lett 5: 1149-1154, 2013.

10. Catto JW, Miah S, Owen HC, Bryant H, Myers K, Dudziec E, Larré S, Milo M, Rehman I, Rosario DJ, et al: Distinct microRNA alterations characterize high- and low-grade bladder cancer. Cancer Res 69: 8472-8481, 2009. 
11. Wu D, Tao J, Xu B, Li P, Lu Q and Zhang W: Downregulation of Dicer, a component of the microRNA machinery, in bladder cancer. Mol Med Rep 5: 695-699, 2012.

12. Han Y, Liu Y, Gui Y and Cai Z: Inducing cell proliferation inhibition and apoptosis via silencing Dicer, Drosha, and Exportin 5 in urothelial carcinoma of the bladder. J Surg Oncol 107: 201-205, 2013.

13. Tao J, Wu D, Li P, Xu B, Lu Q and Zhang W: microRNA-18a, a member of the oncogenic miR-17-92 cluster, targets Dicer and suppresses cell proliferation in bladder cancer T24 cells. Mol Med Rep 5: 167-172, 2012.

14. Zeng S, Yang J, Zhao J, Liu Q, Rong M, Guo Z and Gao W: Silencing Dicer expression enhances cellular proliferative and invasive capacities in human tongue squamous cell carcinoma. Oncol Rep 31: 867-873, 2014.

15. Moyret-Lalle C, Ruiz E and Puisieux A: Epithelial-mesenchymal transition transcription factors and miRNAs: 'Plastic surgeons' of breast cancer. World J Clin Oncol 5: 311-322, 2014.

16. Grelier G, Voirin N, Ay AS, Cox DG, Chabaud S, Treilleux I, Léon-Goddard S, Rimokh R, Mikaelian I, Venoux C, et al: Prognostic value of Dicer expression in human breast cancers and association with the mesenchymal phenotype. Br J Cancer 101: 673-683, 2009.

17. Nieman MT, Prudoff RS, Johnson KR and Wheelock MJ: $\mathrm{N}$-cadherin promotes motility in human breast cancer cells regardless of their E-cadherin expression. J Cell Biol 147: 631-644, 1999

18. Kariko K, Malkowicz S, Li W, Kuo A and Barnathan E: Invasive neoplastic uroepithelial cells express high-levels of urokinase receptor and plasminogen receptor, alpha-enolase. Int J Oncol 3: 1089-1095, 1993.

19. Wu X, Obata T, Khan Q, Highshaw RA, De Vere White R and Sweeney C: The phosphatidylinositol-3 kinase pathway regulates bladder cancer cell invasion. BJU Int 93: 143-150, 2004

20. Su X, Chakravarti D, Cho MS, Liu L, Gi YJ, Lin YL, Leung ML, El-Naggar A, Creighton CJ, Suraokar MB, et al: TAp63 suppresses metastasis through coordinate regulation of Dicer and miRNAs. Nature 467: 986-990, 2010.

21. Vasala K, Pääkkö P and Turpeenniemi-Hujanen T: Matrix metalloproteinase-2 immunoreactive protein as a prognostic marker in bladder cancer. Urology 62: 952-957, 2003.

22. Papathoma AS, Petraki C, Grigorakis A, Papakonstantinou H, Karavana V, Stefanakis S, Sotsiou F and Pintzas A: Prognostic significance of matrix metalloproteinases 2 and 9 in bladder cancer. Anticancer Res 20: 2009-2013, 2000.

23. Wszolek MF, Rieger-Christ KM, Kenney PA, Gould JJ, Silva Neto B, Lavoie AK, Logvinenko T, Libertino JA and Summerhayes IC: A MicroRNA expression profile defining the invasive bladder tumor phenotype. Urol Oncol 29: 794-801.e1, 2011.
24. Wang S, Li Q, Wang K, Dai Y, Yang J, Xue S, Han F, Zhang Q, Liu J and Wu W: Decreased expression of microRNA-31 associates with aggressive tumor progression and poor prognosis in patients with bladder cancer. Clin Transl Oncol 15: 849-854, 2013.

25. Neely LA, Rieger-Christ KM, Neto BS, Eroshkin A, Garver J, Patel S, Phung NA, McLaughlin S, Libertino JA, Whitney D, et al: A microRNA expression ratio defining the invasive phenotype in bladder tumors. Urol Oncol 28: 39-48, 2010.

26. Mongroo PS and Rustgi AK: The role of the miR-200 family in epithelial-mesenchymal transition. Cancer Biol Ther 10: 219-222, 2010.

27. Zhang SL and Liu L: microRNA-148a inhibits hepatocellular carcinoma cell invasion by targeting sphingosine-1-phosphate receptor 1. Exp Ther Med 9: 579-584, 2015.

28. Pan SJ, Zhan SK, Pei BG, Sun QF, Bian LG and Sun BM: MicroRNA-149 inhibits proliferation and invasion of glioma cells via blockade of AKT1 signaling. Int J Immunopathol Pharmacol 25: 871-881, 2012.

29. Ni X, Xia T, Zhao Y, Zhou W, Wu N, Liu X, Ding Q, Zha X, Sha J and Wang S: Downregulation of miR-106b induced breast cancer cell invasion and motility in association with overexpression of matrix metalloproteinase 2. Cancer Sci 105: 18-25, 2014.

30. Khoshnaw SM, Rakha EA, Abdel-Fatah TM, Nolan CC, Hodi Z, Macmillan DR, Ellis IO and Green AR: Loss of Dicer expression is associated with breast cancer progression and recurrence. Breast Cancer Res Treat 135: 403-413, 2012.

31. Faggad A, Kasajima A, Weichert W, Stenzinger A, Elwali NE, Dietel $M$ and Denkert C: Down-regulation of the microRNA processing enzyme Dicer is a prognostic factor in human colorectal cancer. Histopathology 61: 552-561, 2012.

32. Luo Z, Dai Y, Zhang L, Jiang C, Li Z, Yang J, McCarthy JB, She $\mathrm{X}$, Zhang $\mathrm{W}$, Ma J, et al: miR-18a promotes malignant progression by impairing microRNA biogenesis in nasopharyngeal carcinoma. Carcinogenesis 34: 415-425, 2013.

33. Iliou MS, da Silva-Diz V, Carmona FJ, Ramalho-Carvalho J, Heyn H, Villanueva A, Muñoz P and Esteller M: Impaired DICER1 function promotes stemness and metastasis in colon cancer. Oncogene 33: 4003-4015, 2014.

34. Tzamali E, Grekas G, Marias K and Sakkalis V: Exploring the competition between proliferative and invasive cancer phenotypes in a continuous spatial model. PLoS One 9: e103191, 2014.

35. Zhang B, Chen H, Zhang L, Dakhova O, Zhang Y, Lewis MT, Creighton CJ, Ittmann MM and Xin L: A dosage-dependent pleiotropic role of Dicer in prostate cancer growth and metastasis. Oncogene 33: 3099-3108, 2014. 